\title{
Long-Period Fiber Gratings With Overlay of Variable Refractive Index
}

\author{
Ignacio Del Villar, Ignacio. R. Matias, Senior Member, IEEE, and Francisco J. Arregui, Member, IEEE
}

\begin{abstract}
A theoretical analysis is presented of a long-period fiber grating (LPFG) with an overlay of variable refractive index. The highest sensitivity of the resonance wavelengths to variations in the refractive index of the overlay can be optimized. There are two key points for a good design: the selection of an overlay refractive index close to that of the cladding of the LPFG and the overlay thickness. The problem is analyzed with a numerical method based on coupled-mode theory.
\end{abstract}

Index Terms-Coupled-mode analysis, gratings, optical fiber sensors, thin films.

\section{INTRODUCTION}

$\mathbf{L}$ ONG-PERIOD fiber gratings (LPFGs) consist of an index modulation of the refractive index of the core of a singlemode fiber, with a much longer period than fiber Bragg gratings (FBGs). Its particularity, if we compare them with FBGs, is that there is a coupling between the core and the copropagating cladding modes. For this reason, they find application in optical communications [1] and sensors [2].

Regarding the analysis of LPFGs, two different cases have been studied so far. The first case is limited to ambient refractive indexes lower than the cladding. In [3], there is a study with hybrid modes and a uniform azimutal perturbation, whereas in [4], the LP mode approximation is used for arbitrary azimutal perturbation. As the ambient refractive index approaches that of the cladding, the sensitivity of the resonance wavelength to variations of the ambient refractive index is higher. The second case starts when the ambient refractive index exceeds that of the cladding. The core couples with radiation modes [5], [6] and the dependence of the resonance wavelength on the ambient refractive index is not so straightforward. Instead, the resonance depth is more dependent on this parameter for values close to the refractive index of the cladding. In both cases, the region of highest sensitivity is located around the refractive index of the cladding.

Recently, a third case has been analyzed both theoretically and experimentally [7]-[9]. In the previous two cases, the cladding was surrounded by a medium of infinite thickness. Now there is a thin overlay of higher refractive index than the cladding between the cladding and the infinite surrounding

Manuscript received April 29, 2005: revised May 20, 2005. This work was supported by Spanish Ministerio de Ciencia y Tecnologia and FEDER Research Grants CICYT-TIC 2003-00909, and by Gobierno de Navarra and FPU MECD Grant.

The authors are with the Electrical and Electronic Engineering Department, Public University of Navarra, Pamplona 31006, Spain (ignacio.delvillar@unavarra.es; natxo@unavarra.es; parregui@unavarra.es).

Digital Object Identifier 10.1109/LPT.2005.853283

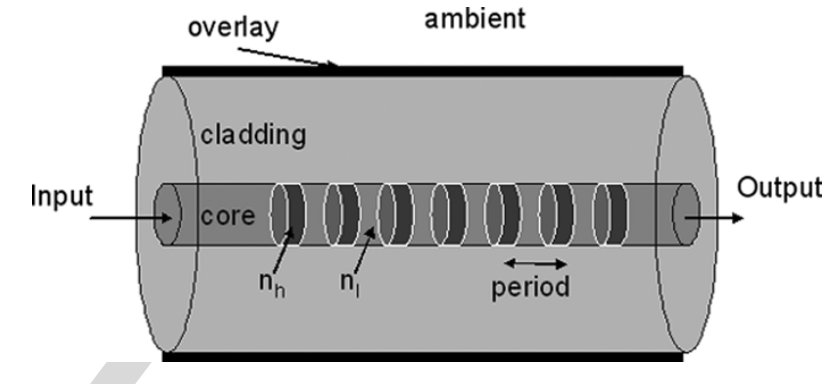

Fig. 1. LPFG structure with overlay on the cladding.

media. One of the cladding modes will be guided by the overlay if it is thick enough [8]. This causes a reorganization of the effective indexes of the modes of the cladding. As a result, there are important variations of the Bragg condition, which leads to dramatic shifts of the resonance wavelengths if we work around the thickness value where there is a transition to guidance of a mode in the overlay.

This is the first time, to our knowledge, that variations of the overlay refractive index are analyzed, which has a different behavior than the variation of the overlay thickness. It is proved that shifts of about $50 \mathrm{~nm}$ can be obtained, provided a good design is selected. This could be applied toward development of practical tunable optical filters with a wider wavelength range than previous designs [10]. There exist materials with a variation of refractive index, which are suitable for this design such as liquid crystals [11] and magnetooptic ones. However, the nanodeposition of these materials on the cladding of an LPFG is not an easy task. So far, developed experiments are based on Langmuir Blodgett [7] and the electrostatic self-assembly method [9]. Consequently, typical refractive index values in the range between 1.55 and 1.8 have been analyzed. Other applications are the improvement of sensors based on materials of variable refractive index, and the improvement of the performance of tunable microfluidic optical fibers [12]. Another remarkable novelty in this work is that the hybrid mode analysis is used instead of the LP mode approximation.

\section{THEORY}

Coupled-mode theory has proved to be a powerful tool for simulation of LPFG structures [3], [4]. The problem analyzed in this work (see Fig. 1) presents four layers, and follows the same steps as in the three-layer model of [3]. For the sake of simplicity, the structure simulated presents no azimutal variation of the perturbed index profile after exposure to ultraviolet radiation. In this way, there are only interactions between the core mode $H E_{11}$, and $H E_{1 j}$ and $E H_{1 j}$ modes of the cladding. Once 
the HE and EH modes are calculated, the self- and cross-coupling coefficients are obtained and they are introduced in the coupled-mode differential equations.

When the goal is just to see the displacement of the resonance wavelengths, alternative solutions to the application of the four steps can be used.

The first one is the calculation of the resonance wavelength with the Bragg condition

$$
\beta_{11}(\lambda)-\beta_{1 j}(\lambda)=\frac{2 \pi}{\Lambda}
$$

where $\beta_{11}$ and $\beta_{1 j}$ are the propagation constants of the core and the $j$ cladding modes, respectively, and $\Lambda$ is the period of the grating. This produces inexact results. However, if the modified first-order Bragg condition is applied, errors are lower than $0.1 \%$ [4]

$$
\beta_{11}(\lambda)+s_{0} \zeta_{11,11}(\lambda)-\left(\beta_{0 j}(\lambda)+s_{0} \zeta_{1 j, 1 j}(\lambda)\right)=\frac{2 \pi}{\Lambda}
$$

where $\varsigma_{01,01}$ and $\varsigma_{0 j, 0 j}$ are the self-coupling coefficients of the core and the $j$ cladding modes, and $s_{0}$ is the coefficient of the first Fourier component of the grating function $S(z)$. This reduces the computational effort in a great manner.

\section{ANALYSIS OF LPFG STRUCTURES With DEPOSITION OF AN OVERLAY}

A commercial LPFG is selected for the analysis performed in this section. A modulation of the core refractive index is induced in a Corning Flexcore fiber like that of [3]. The parameters of the LPFG are: core diameter of $5 \mu \mathrm{m}$, cladding diameter $125 \mu \mathrm{m}$, core refractive index 1.4573 , cladding refractive index 1.45 , period of the grating $276 \mu \mathrm{m}$, and length of the grating $25 \mathrm{~mm}$. The modulation is considered sinusoidal. Consequently the apodization factor $\sigma(z)=s_{0}=s_{1}=1$. The amplitude of the modulation is $2.7 \times 10^{-4}$. The ambient refractive index is assumed to be one (air). The notation used for the modes is $\mathrm{HE}_{1,1}$ for the core mode, $\mathrm{HE}_{1,2}$ for the first $\mathrm{HE}_{1, j}$ cladding mode, $\mathrm{HE}_{1,4}$ for the second $\mathrm{HE}_{1, j}$ cladding mode, and so on; $\mathrm{EH}_{1,3}$ for the first $\mathrm{EH}_{1, j}$ cladding mode, $\mathrm{EH}_{1,5}$ for the second $\mathrm{EH}_{1, j}$ cladding mode, and so on.

In [9], it was experimentally and theoretically proved with the LP mode approximation that if an overlay of higher refractive index than the cladding is deposited on an LPFG, as the thickness of the overlay increases, cladding modes shift their effective index to higher values. When the overlay is thick enough, one of the cladding modes is guided by the overlay. Provided the waveguide is lossless, the highest effective index mode (lowest order cladding mode) becomes guided. This causes a reorganization of the effective index of the rest of modes. Higher order cladding modes than the one that is guided by the overlay will shift their effective index value toward the effective index of the immediate lower order cladding mode of the LPFG without overlay. As more material is deposited, the effective index distribution before deposition is recovered. With the hybrid mode analysis performed in this work, the idea remains the same. When there is no azimutal perturbation $\mathrm{LP}_{0, j}$ modes correspond with $\mathrm{HE}_{1, j}$ modes. Consequently, each $\mathrm{HE}_{1, j}$ mode will shift its

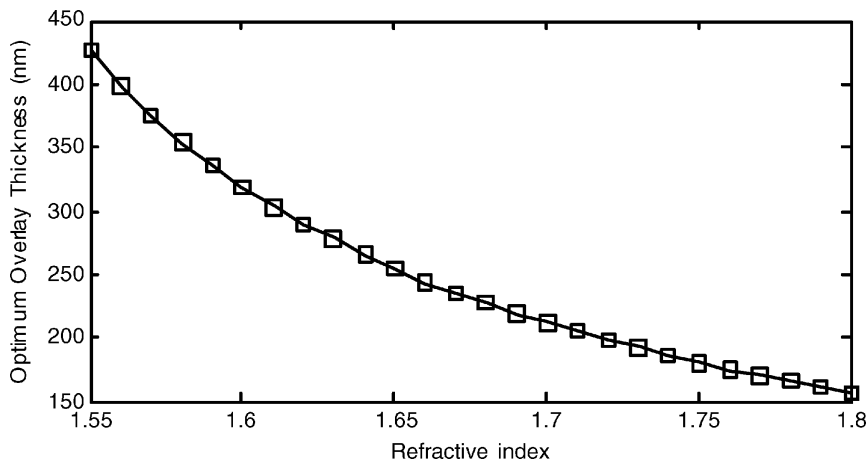

Fig. 2. OOT as a function of the overlay refractive index for the $\mathrm{HE}_{1,16}$ resonance.

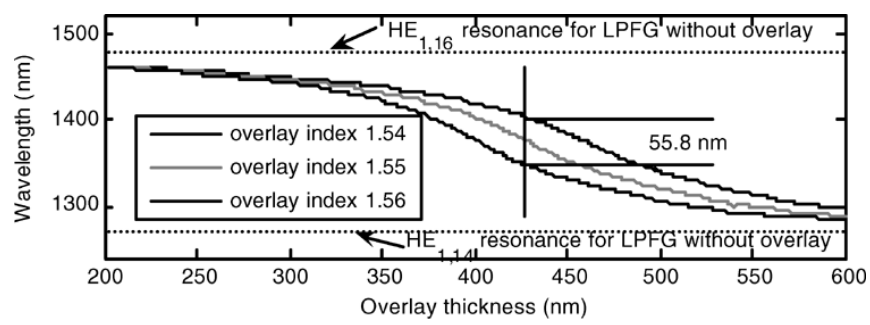

(a)

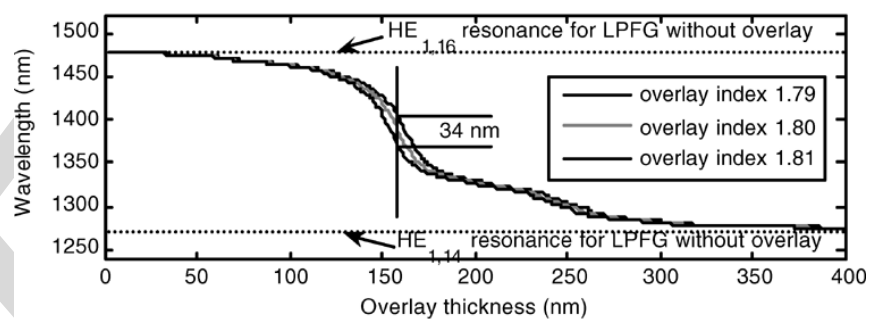

(b)

Fig. 3. $\mathrm{HE}_{1,16}$ resonance wavelength as a function of the overlay thickness for (a) 1.55 and two close refractive indexes: 1.54 and 1.56. (b) 1.8 and two close refractive indexes: 1.79 and 1.81 .

effective index to that of the immediate lower order $\mathrm{HE}_{1, j}$ mode of the LPFG without overlay.

The immediate consequence of the shift in effective index is that it leads to a displacement in all the attenuation bands. The attenuation band corresponding with the $\mathrm{HE}_{1,16}$ (respectively, $\mathrm{LP}_{0,9}$ mode) shifts the wavelength to that of the $\mathrm{HE}_{1,14}$ (respectively, $\mathrm{LP}_{0,8}$ mode); the same is true for the $\mathrm{HE}_{1,14}$ (respectively, $\mathrm{LP}_{0,8}$ mode) that shifts the wavelength to the attenuation band of the $\mathrm{HE}_{1,12}$ (respectively, $\mathrm{LP}_{0,7}$ mode), and so forth. Furthermore, there are optimum overlay thickness (OOT) values where the central wavelength shift as a function of the ambient refractive index is highest. In Fig. 2, the OOT of $\mathrm{HE}_{1,16}$ resonance is represented as a function of the overlay refractive index. For higher refractive indexes, the OOT trends to lower values because the guidance in the overlay starts sooner for higher contrast of indexes between the cladding and the overlay.

To understand the influence of the overlay refractive index on the shift of the resonance wavelength, the two extreme values of Fig. 2 (1.55 and 1.8) are analyzed in Fig. 3. In Fig. 3(a), the $\mathrm{HE}_{1,16}$ resonance wavelength is represented as a function of the overlay thickness for an overlay refractive index 1.55 and for two close refractive index values: 1.54 and 1.56 . The same is 


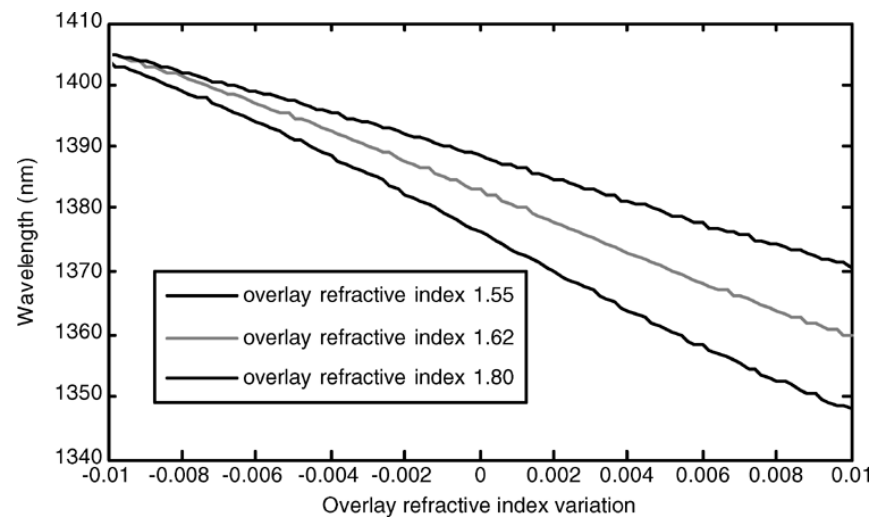

Fig. 4. $\mathrm{HE}_{1,16}$ resonance wavelength as a function of the relative variation of the overlay refractive index for three indexes: $1.55,1.62$, and 1.8 .

done in Fig. 3(b) for 1.8 and two close refractive indexes: 1.79 and 1.81. In all cases, the $\mathrm{HE}_{1,16}$ resonance wavelength shifts to that of the $\mathrm{HE}_{1,14}$ resonance of an LPFG without overlay, as expected. However, the most interesting conclusion is that for a fixed overlay thickness, the wavelength shift produced by variation of the overlay refractive index is much higher for overlay refractive indexes close to the cladding of the LPFG.

This is confirmed in Fig. 4 by comparing the wavelength shift for overlay refractive index variations of \pm 0.01 . Three different refractive indexes are analyzed: $1.55,1.62$, and 1.8 with overall wavelength shifts $34,45.4$, and $55.8 \mathrm{~nm}$, respectively. This behavior is opposite to the wavelength shift as a function of the overlay thickness variation for a fixed refractive index [8]. The reason is that, though the wavelength shift as a function of the overlay thickness is more abrupt for high overlay refractive indexes, the variation of the plots obtained in Fig. 3 is smaller [Fig. 3(b)] compared to those of close refractive indexes to that of the cladding [Fig. 3(a)].

\section{CONCLUSION}

In this letter, the effect of variation in the refractive index of an overlay deposited on the cladding of an LPFG with a refractive index higher than that of the LPFG has been analyzed. To this purpose, the theory of mode perturbation induced by thin overlay on the cladding of an LPFG [8] has been applied. This is the first time coupled-mode analysis with hybrid modes is used instead of LP mode approximation. Shifts of more than $50 \mathrm{~nm}$ in the attenuation bands of the transmission spectrum can be obtained provided an adequate design is selected. For a specific LPFG and ambient refractive index, it is critical to select an overlay thickness that optimizes the wavelength shift for a specific overlay refractive index. The closer the refractive index is to the cladding of the LPFG, the wider is the wavelength shift of the resonances as of function of the relative variation of the overlay refractive index. This behavior is opposite to the effect of varying the overlay thickness, where the wavelength shift is wider for higher overlay refractive indexes.

In conclusion, if the design is aimed for a material of variable thickness (i.e., a material that expands if temperature is increased), the best choice is a high overlay refractive index [8], whereas, in this letter, it has been proved that for a material of variable refractive index the best choice is a refractive index close to that of the cladding of the LPFG.

This analysis could find application in sensors and, due to the wide spectral shift, in optical switches and filters.

\section{REFERENCES}

[1] A. M. Vengsarkar, P. J. Lemaire, J. B. Judkins, V. Bhatia, T. Erdogan, and J. E. Sipe, "Long-period fiber gratings as band rejection filters," $J$. Lightw. Technol., vol. 14, no. 1, pp. 58-65, Jan. 1996.

[2] S. W. James and R. P. Tatam, "Optical fiber long-period grating sensors: Characteristics and application," Meas. Sci. Technol., vol. 14, pp. R49-R61, 2003.

[3] T. Erdogan, "Cladding-mode resonances in short- and long-period fiber gratings filters,” J. Opt. Soc. Amer. A, vol. 14, pp. 1760-1773, 1997.

[4] E. Anemogiannis, E. N. Glytsis, and T. K. Gaylord, "Transmission characteristics of long- period fiber gratings having arbitrary azimutal/radial refractive index variation," J. Lightw. Technol., vol. 21, no. 1, pp. 218-227, Jan. 2003.

[5] D. B. Stegall and T. Erdogan, "Leaky cladding mode propagation in long-period fiber grating devices," IEEE Photon. Technol. Lett., vol. 11, no. 3, pp. 343-345, Mar. 1999.

[6] Y. Koyamada, "Numerical analysis of core-mode to radiation-mode coupling in long-period fiber gratings," IEEE Photon. Technol. Lett., vol. 13, no. 4, pp. 308-310, Apr. 2001.

[7] N. D. Rees, S. W. James, R. P. Tatam, and G. J. Ashwell, "Optical fiber long-period gratings with Langmuir-Blodgett thin-film overlays," Opt. Lett., vol. 27, pp. 686-688, 2002.

[8] I. Del Villar, I. R. Matias, F. J. Arregui, and P. Lalanne, "Opitmization of sensitivity in long period fiber gratings," Opt. Express, vol. 13, pp. 56-69, 2005.

[9] I. Del Villar, M. Achaerandio, I. R. Matias, and F. J. Arregui, “Deposition of an overlay with electrostactic self-assembly method in long period fiber gratings," Opt. Lett., vol. 30, pp. 720-722, 2005.

[10] I. Del Villar, I. R. Matias, F. J. Arregui, and R. O. Claus, "Analysis of one-dimensional photonic bandgap structures with a liquid crystal defect toward development of fiber-optic tunable wavelength filters," Opt. Express, vol. 11, pp. 430-436, 2003.

[11] Y. Jeong, H. R. Kim, S. Baek, Y. Kim, Y. W. Lee, S. D. Lee, and B Lee, "Polarization-isolated electrical modulation of an etched long-period fiber grating with an outer liquid-crystal cladding," Opt. Eng., vol. 42, pp. 964-968, 2003.

[12] P. Mach, M. Dolinski, K. W. Baldwin, J. A. Rogers, C. Kerbage, R. S. Windeler, and B. J. Eggleton, "Tunable microfluidic optical fiber," Appl. Phys. Lett., vol. 80, pp. 4294-4296, 2002. 